\title{
An SDL (Simple Description Language) Analyzer
}

\author{
Robert J. Borrmann, Ph.D. \\ Professor of Electrical and Computer Engineering \\ Manhattan College \\ New York City
}

\begin{abstract}
Second-year students in Manhattan's four-year Electrical Engineering and Computer Engineering programs are introduced to digital circuits in a one-semester 3-credit course ELEC-229. This course includes a laboratory component in which students design and breadboard simple circuits. While the course includes preliminary coverage of VHDL (Very High Speed Integrated Circuits Hardware Description Language), the author believes that an easier introductory language and analysis tool, designed to parallel the student's progress in learning digital concepts, can help them gain confidence and familiarity with the strengths and weaknesses of digital simulation.
\end{abstract}

The SDL Analyzer described in this paper uses the power of Visual Basic graphics to provide simple logic simulation capabilities for small circuits of the complexity typically found in introductory courses. It is designed as a tutorial aid for students in digital analysis and design courses, and is based on the author's experience teaching the material in the college classroom. Compared with standard VHDL simulators, SDL provides a simpler user interface and reduced capabilities. Students can operate it interactively on their own computers, and study circuit operations step-by-step at their own pace. They can stimulate the circuit interactively, and view the results of analysis, either in tabular form or on the schematic drawn by the analyzer. All results can be saved to disk or printed.

This paper describes the SDL language, the SDL analyzer, and includes comparisons of SDL with ABEL and with VHDL, and the author's experiences using SDL in the classroom.

\section{Introduction.}

It is an accepted principle that students' learning of engineering concepts can be aided by providing them with suitable computer simulation tools. In their first course in digital systems, engineering students are introduced to logic gates, binary and hexadecimal numbers, flipflops, registers, and memory. A number of general-engineering tools are available, such as MATLAB ${ }^{1}$ and $\mathrm{MathCad}^{2}$, but these require some programming by the user to simulate digital circuits. Some programs are tailored to digital simulation, at least in part. This list includes $\mathrm{ABEL}^{3}$, Electronics Workbench ${ }^{4}$, LogicWorks ${ }^{5}$, PSPICE $^{6}$, $\mathrm{VHDL}^{7}$, and others. These packages vary in a number of respects, including power, cost, complexity, suitability for classroom use, and schematic capture. Those that rely 
primarily on text input define hardware description languages (HDLs).

After a hiatus of over fifteen years, I recently returned to teaching introductory digital systems, and saw the same pedagogical attractiveness of simulation as in the past, but a drastically changed computing landscape. Personal computers with extensive graphics capabilities now abound. Hardware description languages and electronic simulation packages are well-standardized and widely accepted. However, the fashion towards increasing power in these areas mirrors recent software trends towards increasing size and complexity, threatening to drown the beginning student in a sea of detail that obscures the digital principles that she needs to understand. The SDL Analyzer is the culmination of several earlier efforts, ranging from 30-year-old FORTRAN punch-card programs, to later assembly-language and QBASIC programs. Their common objective was to provide students with a means of verifying their analytical and design work in order to reinforce their understanding of their course material. Since the new program uses custom-formatted text files as inputs, it defines a language. In this paper, both the program and the language are referred to simply as $S D L$.

I began to develop SDL in Fall 1999 to satisfy the following criteria:

-Simplicity. The program should present a simple intuitive graphical user interface, and should require a minimum of programming knowledge on the part of the student.

-Size. The program should be small and easily installable on students' own computers.

- Familiarity. The program should use common terminology that echoes the vocabulary used in the course and in the textbook.

- Cost/Benefit. The program should offer sufficient functionality to make worthwhile the investment of student time needed to learn it.

- Migration. The program should lay a foundation for migration to more powerful standard tools such as VHDL.

As of the time of this writing, program development has continued for approximately 15 months and reflects improvements prompted by experiences with teaching students over that period.

II. SDL Capabilities In Brief.

- Simple or hierarchical digital circuits consisting of gates, multiplexers, decoders, latches, flipflops, registers, memories, etc.

- Combinational, synchronous sequential (Mealy and Moore types), and asynchronous circuits.

-Structural, behavioral, and functional analyses.

- Multibit signals; binary, decimal, and hexadecimal arithmetic.

- Schematic output; tabular and interactive analyses; macros and tests.

Some limitations of SDL are:

- Limited treatment of propagation delays, race, amd hazard conditions.

- Limited circuit size (e.g., no more than 100 gates or 500 variables).

- Fixed library of components.

- No schematic capture.

\section{Description of SDL.}

The following paragraphs describe more fully and illustrate some of SDL's capabilities.

Inputs. SDL is a hardware description language (HDL). SDL accepts text files as 
inputs. Each file describes one or more circuits, and contains statements such as

d)

$$
\mathbf{z}=\operatorname{AND}(\mathrm{x}, \mathrm{y}) \quad \mathrm{and}=\mathrm{OR}(\mathrm{a}, / \mathrm{b}, / \mathrm{c},
$$

which the student can easily identify with the AND- and OR-gates he studies in the course. The variable names (z, x, y, g, a, etc.) are user-selectable. Each statement occupies one line. The slash (/) preceding variable names $\mathbf{b}$ and $\mathbf{c}$ denotes the one's complement (the NOT function) of the variable. The functions AND and OR, along with several dozen others, are built into the program. Gates can have any number of inputs, limited only by considerations of possible crowding on schematic drawings..

In SDL circuit descriptions, a minimum of "boilerplate" is required. The complete file describing a one-bit half-adder consisting on one exclusive-OR gate and one AND gate is shown in Listing 1. (In this and subsequent listings, SDL keywords are shown in boldface for purposes of explanation in this paper, but boldfacing is not a part of the SDL language.) The similarities between SDL syntax and VHDL syntax are obvious.

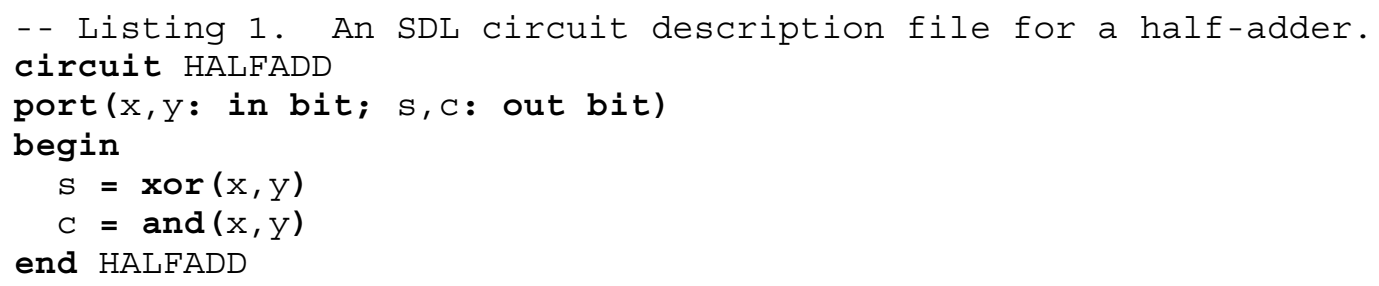

Outputs. When the student opens a circuit description file in the SDL analyzer, he sees a copy of it in the program's "circuit" window, and several command buttons representing available action options. Clicking on the "Truth Table" command button produces the truth table of the circuit in the "results" window. Clicking on the "Interactive" command button puts the program into its interactive mode (discussed later). Selecting a menu command displays the circuit's schematic in another window.

Hierarchical Circuits. SDL's simple syntax extends to hierarchical circuits as well. For example, Listing 2 shows the SDL circuit description file defining a full-adder in terms of two half-adders and an OR gate.

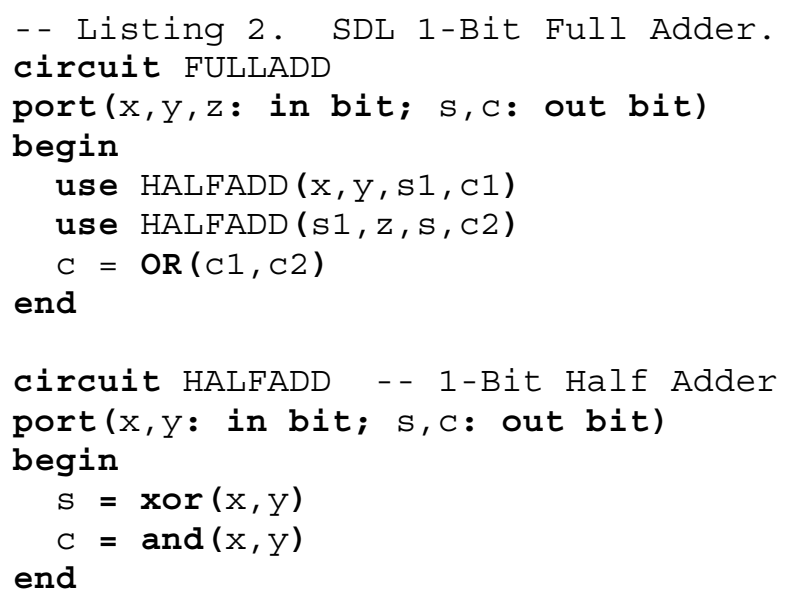


The SDL keyword use links the parent full-adder circuit to the half-adder subcircuit. This keyword is followed by the subcircuit's name HALFADD and (in parentheses) by the names of the communicating variables. As in VHDL port maps, these function arguments must agree in type and number with the input and output variables of the subcircuit as declared in the subcircuit's port line. Unlike VHDL, however, no component predeclaration is needed. The SDL user can interactively make any circuit of a hierarchical circuit file the "current" circuit, and therefore can study the characteristics of each subcircuit, as well as of the parent circuit, with equal ease.

Statement Order and Error Detection. SDL statements are executed sequentially, so that variables $c 1$ and $c 2$ must be evaluated before the statement $\mathrm{c}=\mathbf{O R}(\mathrm{c} 1, \mathrm{c} 2)$ can be executed. Therefore, the order of statements in an SDL file is significant. In Fig. 2, the two preceding use statements produce $c 1$ and $c 2$ as outputs, so that the OR statement can be executed successfully. Mis-ordering of statements generally results in unknown value of input (UVI) errors when the circuit file is analyzed, if the user has opted to treat this condition as an error. When SDL finds an error in an SDL file, it reports it to the user, and highlights the offending line in the circuits description window. Contextsensitive debugging help is offered in the built-in editor to assist in making rapid corrections to the file.

By default, SDL also checks for contention in the circuit. Ordinarily contention is caused by a coding error in the circuit file (e.g., two gate outputs are connected together), but it can also be caused by a circuit operational error (e.g., two tri-state buffers feeding the same bus are mistakenly enabled at the same time).

Synchronous Sequential Circuits. Sequential circuits require two additional parts of the SDL language. First, a state line is needed to declare which variables are state variables (i.e., feedback variables) in the system. The state line also specifies which circuit inputs are clock variables to the system. SDL uses the state information in setting up the table columns for producing the state transition table for the system, and for the interactive mode of operation.

The second addition includes flipflop, register, and regfile functions, which model various types of latches, flipflops, registers, and memory devices. Different types of flipflops or registers are represented by the same flipflop and register functions, with different types of labeled arguments included. For example, the circuit file in Listing 3 describes a Mealy-type synchronous sequential circuit using two Type-D flipflops, based on a design problem in a Digital Systems textbook ${ }^{8}$. Bits $a$ and $b$ are the flipflop outputs. Both flipflops are edge-sensitive (denoted by the keyword eclk=), and both are type-D (denoted by the keyword $\mathbf{d}=$ ). Other flipflop types use other keywords, such as $\mathbf{J}=$ and $\mathbf{K}=$ for JK-flipflops, $\mathbf{S}=$ and $\mathbf{R}=$ for $\mathrm{SR}$ latches, etc.

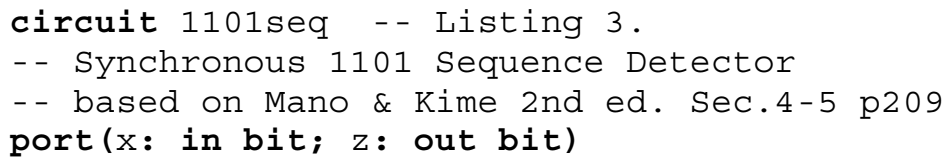




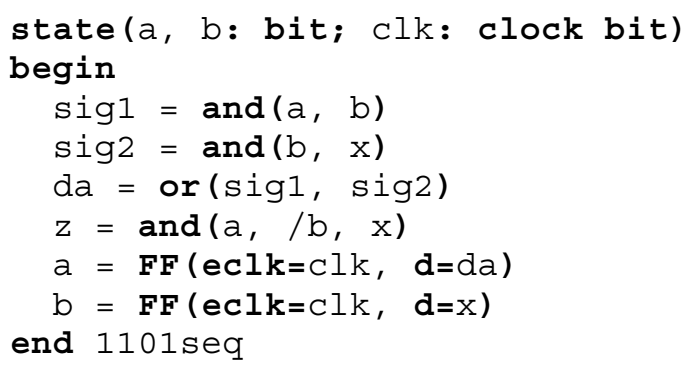

Statement Order Again. In synchronous sequential circuits, the body of the file (between begin and end) can be considered to be divided into two parts. The combinational part of the circuit must be described in the first part of the file. All ff and reg statements must appear in the second part of the file. Finally, all the inputs needed by the ff and reg functions in the second part must be circuit inputs, or must be evaluated in the combinational statements of the first part. These requirements guarantee the concurrency of the state changes in the circuit.

Multibit Variables. In SDL, variables typically do not need declarations unless they are circuit inputs or outputs (these declarations occur in the port line), or unless they are feedback variables (these declarations occur in the state line). In port and state lines, variables are declared to be multibit using the bitwidth specifier forms bit(n..0), nibble, or byte instead of bit. Other variables generally need no declarations. Their bitwidths are implied when they are expressed as outputs of gate functions. When gate inputs are multibit variables, their outputs are also multibit, and each gate function represents an array of gates.

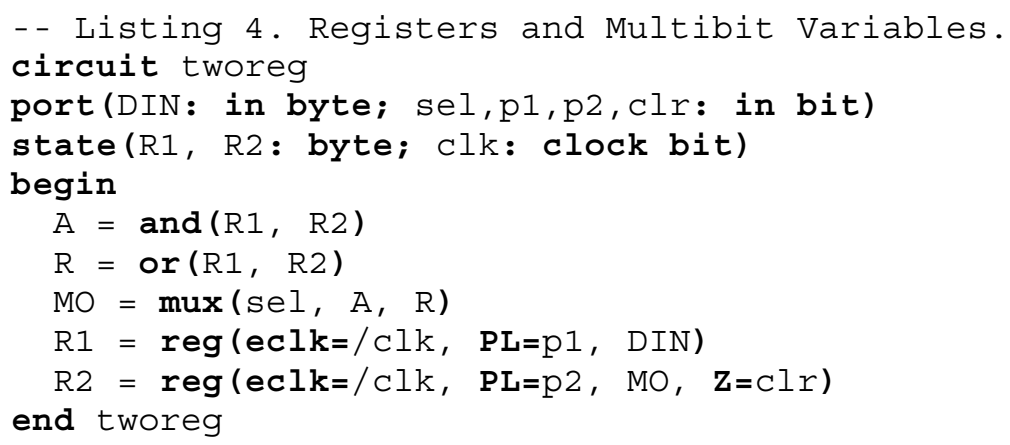

For example the and function in Listing 4 represents an array of eight and gates (because each input R1 and R2 is a byte), and the or function represents an array of eight or gates for the same reason. Gate outputs $A$ and $R$ have bitwidths of 8. The 8-bit 2-way multiplexer mux selects one of the gate outputs $(A$ or $R$ ) for possible input to register R2. Both R1 and R2 are declared to be eight bits wide in the state line. Both registers are synchronously clocked on the negative edge of clk. R1 is parallel-loaded from input bus DIN whenever control input $p 1$ is one. R2 is parallel loaded from the multiplexer output $M O$ whenever control input $p 2$ is one, and is cleared whenever control input $c l r$ is one. 
Memory modules can be modeled as register files, i.e., arrays of registers. The statement MEM: regfile $(16,8)$ declares MEM to be a $16 \times 8$ memory array (16 words, each word 8 bits wide). Individual words in the array are accessed by literal address (e.g., MEM'7) or by symbolic address (e.g., MEM'addr, where $a d d r$ is the circuit variable carrying the desired memory address). Memories with various read and write control structures can be modeled by using SDL reg functions. For example, Listing 5 shows an SDL circuit file describing a $16 \times 8$ memory module, with data input bus DIN, data output bus DOUT, address input ADRS, chip select CS, and read/write input bit RW.

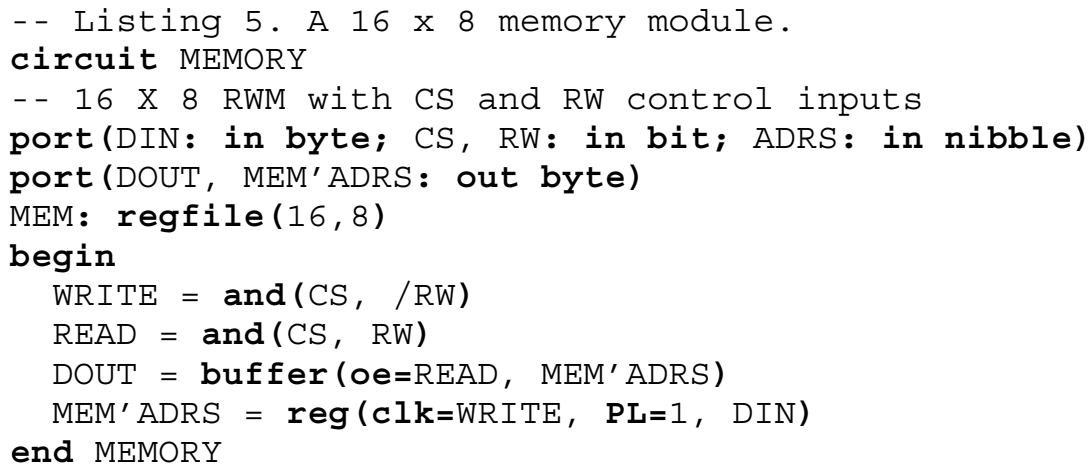

The internal control signal WRITE is active (high) only when CS is high and RW is low; internal control signal READ is active (high) only when CS is high and RW is high. The reg statement causes a write operation (parallel load $\mathbf{P L}=$ ) from input bus DIN to the word at location $A D R S$ when WRITE is active. The oe= part of the buffer statement models the tri-state aspect of the memory. It enables the memory word at address $A D R S$ to appear at the output pins DOUT when READ is active; otherwise the DOUT bus is in its high-impedance state.

Binary and hexadecimal numbers. Numerical values can be specified as signed or unsigned numbers, in binary, octal, decimal, or hexadecimal form. The maximum bitwidth for numbers, as for variables, is 30 bits. The user can specify the output format she wants by using declaration statements, with keyletters $\mathbf{b}, \mathbf{o}, \mathbf{d}, \mathbf{h}$, and $\mathbf{s}$ following the bitwidth specifiers. Variables can also be declared as text. An example SDL file is shown in Listing 6.

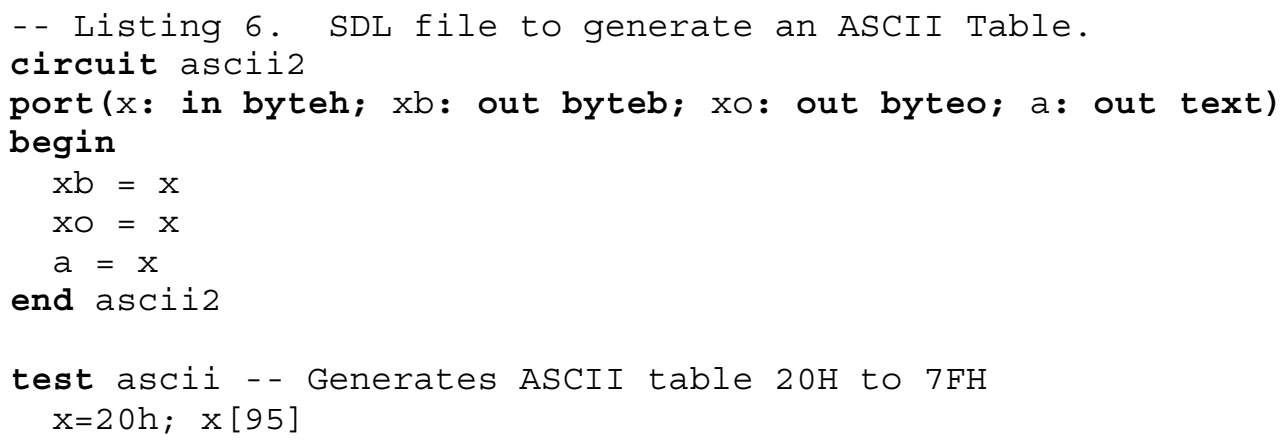

When this file's test macro is run, SDL automatically generates an ASCII table of characters, showing their numeric codes in unsigned hexadecimal, binary, and octal. A 
fragment of this table is shown in Listing 7.

\begin{tabular}{|c|c|c|c|c|}
\hline \multicolumn{5}{|c|}{ Beginning test ascii } \\
\hline $\mathrm{X}$ & $\mathrm{XB}$ & $\mathrm{XO}$ & A & ascii2 \\
\hline $20 \mathrm{~h}$ & $00100000 \mathrm{~b}$ & 0400 & & $x=20 h$ \\
\hline $21 \mathrm{~h}$ & $00100001 \mathrm{~b}$ & 0410 & $!$ & $\mathrm{x}[95]$ \\
\hline $22 \mathrm{~h}$ & $00100010 \mathrm{~b}$ & 0420 & $"$ & \\
\hline $23 \mathrm{~h}$ & $00100011 \mathrm{~b}$ & 0430 & \# & \\
\hline $24 h$ & $00100100 \mathrm{~b}$ & 0440 & $\$$ & \\
\hline $25 \mathrm{~h}$ & $00100101 \mathrm{~b}$ & 0450 & $\%$ & \\
\hline $26 \mathrm{~h}$ & $00100110 \mathrm{~b}$ & 0460 & $\&$ & \\
\hline
\end{tabular}

The Interactive Mode. Students learn best by doing. When solving a problem by hand, the student naturally sees his answer unfold in a gradual manner. It is appropriate that a simulation tool designed for learning should allow a student to see intermediate results, too. That is one role of SDL's interactive mode, which produces tabular output or schematic (graphical) output, or both. In the tabular form all input, output, and state variables are arranged in columns in the results window, as in the ASCII table of Fig. 7. SDL's paradigm is user event driven. To change the value of an input or state variable, the user mouse-clicks on its name in the window's footer area. The analyzer then displays the resulting outputs from the circuit on a new line in the window. To examine the values of internal circuit variables, the user can either (a) add their names to the port line as circuit outputs, (b) enter a "manual command" to interrogate the analyzer, (c) view the schematic drawing (described below), or (d) put the analyzer into its trace mode when making its analysis. This latter option generates voluminous information concerning the values SDL finds for all circuit variables as it progresses through the circuit description line by line. Trace information can be saved or printed if desired.

Graphical Output. An SDL user can produce a schematic drawing from the circuit description file by issuing a command from the program's menu. An example schematic, produced from the SDL file of Listing 3, is shown in Fig. 1. This example includes the optional labels at each node showing both the names and values of the variables. The slash notation (e.g., 0/1) shows the variable's value and bitwidth. In this example, all variables are binary (i.e., bitwidth $=1$ ).

In SDL's graphical interactive mode, the user clicks on any input node (here, $A$ or $C L K$ ) to change the value of the variable. The analyzer instantly recomputes all variables' values, and displays them on the schematic. The user can also change state variables $(A$ and $B$ ) in this way. This allows her to put the circuit into any desired initial state. Stimuli can also be provided by keyboard commands. 


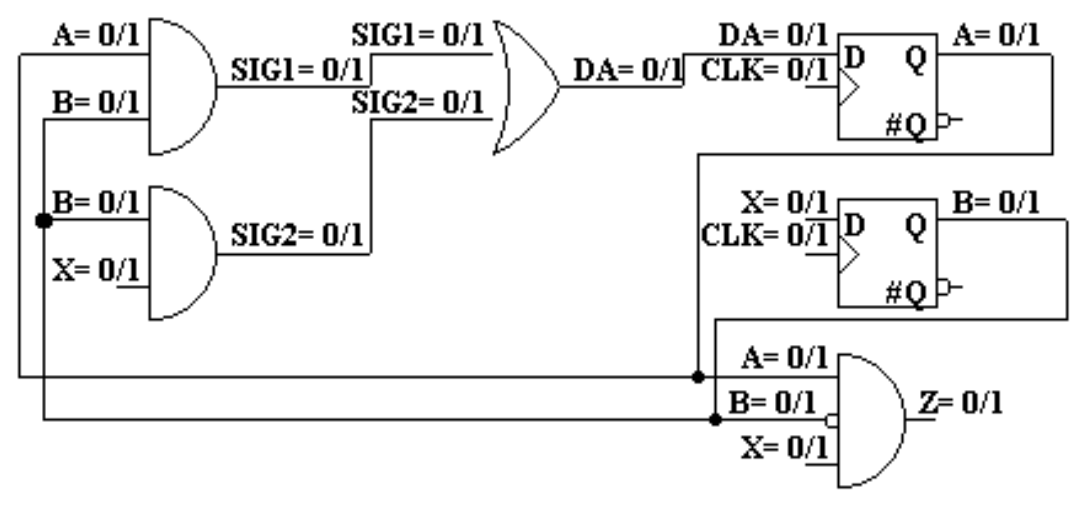

Fig. 1. Circuit schematic produced from the circuit file of Listing 3.

I encourage students to use SDL's graphical interactive mode to help them learn the characteristics of flipflops and registers. For this purpose, I provide them with some very simple SDL files (each one containing only one component, such as a JK-flipflop). I instruct the students to display the component in enlarged form in the drawing window, and then to exercise the circuit. Each student stimulates the circuit in any way he wishes, thereby creating his own "what if" scenarios. This allows him to learn the flipflop's characteristics experimentally. At the end of this exercise, the student can print or save the results listbox, which has silently recorded the sequence of stimuli and responses. This tabular record provides self-authored notes which the student can use at any later time to refresh his memory on details of that flipflop's operation.

In a similar way, a student can also drill himself on common register operations, such as parallel-loading, shifting, and counting, by using simple one-register circuit description files.

Another use of SDL's graphical output, besides its interactive one, is that of documentation. Students can document their simulation results with both the text of the circuit description file, and the schematic drawing of the circuit. An added benefit is in the detection of logical errors. A student can better recognize from a schematic when the SDL analyzer is interpreting her circuit description file in a way she did not intend.

Asynchronous Circuits. The simplest asynchronous circuits are those with only local feedback, where the feedback is confined within elementary latches, flipflops, registers, and memories. SDL includes a built-in functions ff and reg to handle them. Coverage includes clocked and unclocked SR latches, and flipflops and registers with synchronous and asynchronous inputs. When the output of a circuit is uncertain (as when an SR latch makes a transition from its forbidden state to its memory state) SDL displays the value of the output variable with a question mark (?) to signify it is unknown.

For successful simulation by SDL of a general sequential circuit, it is necessary for the user to include all state (i.e., feedback) variables in the SDL file's state line. If this is not done, SDL will find unknown values in inputs and/or outputs during the course of its analysis. The user is guided by the program's outputs to identify feedback variables, 
which he can then add to the state line. Successive refinements should eventually result in a file that can be analyzed completely.

To analyze circuits such as asynchronous ripple counters, or to detect negative feedback situations such as Fig. 2 which, in the absence of propagation delay effects, can have no static solution, SDL iterates its analysis. After each new input stimulus, SDL computes the values of all circuit variables. If it finds that one or more of them has changed in response to the stimulus, it recomputes them all again with the same input stimulus but with the most recent values of the state variables. It is only when the values of all circuit variables agree with the results for the previous iteration that the values are accepted as correct. The maximum number of iterations is user-selectable from 1 to 50, where 1 means effectively that iterations are not done. If no consistent result is found after the maximum number of iterations, the analyzer reports an ITR (iteration) failure to the user.

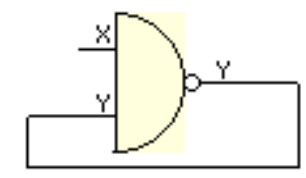

Fig. 2. A simple circuit having no static solution when $x=1$.

\section{Comparisons With Other HDLs.}

It may be instructive to compare SDL with other HDLs. These comparisons will focus on simplicity and small size, since those were primary design criteria for SDL. An ABEL full adder ${ }^{9}$ can be described as in Listing 8:

“Listing 8. A Full Adder in ABEL.

module adder

Title 'full adder cell'

Declarations

A PIN;

B PIN;

Cin PIN;

S PIN istype 'com'; "combinational output

Cout PIN istype 'com';

\section{Equations}

$\mathrm{S}=\mathrm{A}$ \$ B \$ Cin; "sum output

Cout $=$ A \& B \# A \& Cin \# B \& Cin; "carry out

end adder

The corresponding SDL description appears in Listing 9:

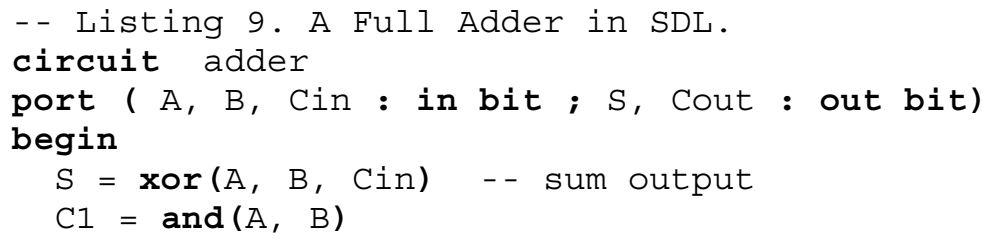




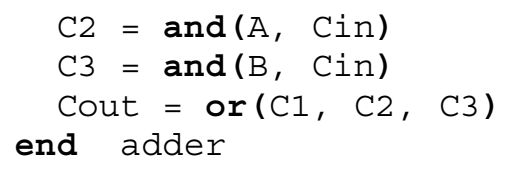

Both files are comparable in size and simplicity, but SDL's xor, and, and or functions are probably more intuitive to digital systems students than ABEL's $\$, \boldsymbol{\&}$, and \# operators.

Like ABEL, SDL allows truth tables as module descriptors, as exemplified in Listing 10.

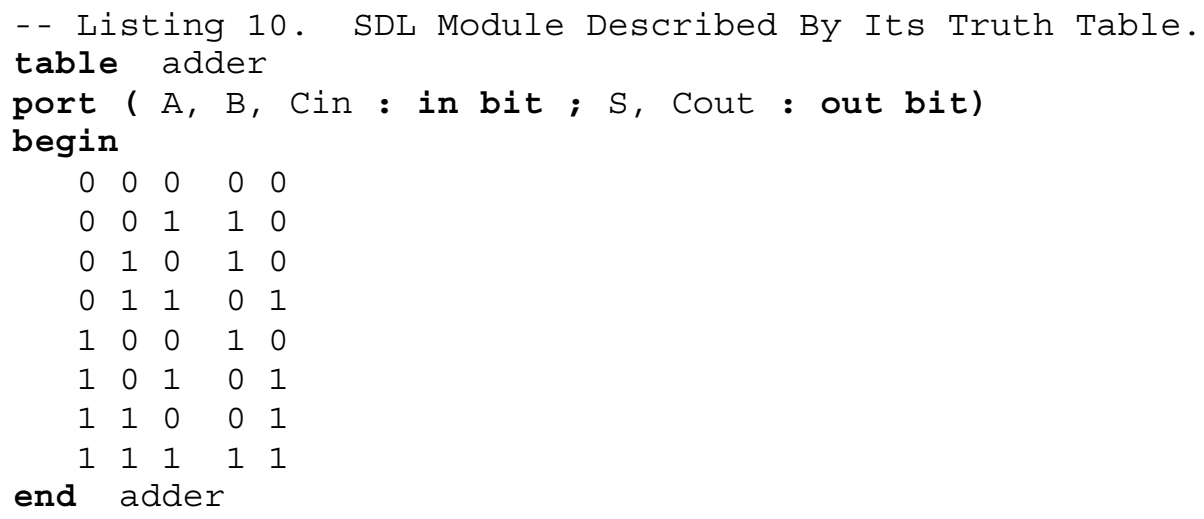

The same module in ABEL is given in Listing 11:

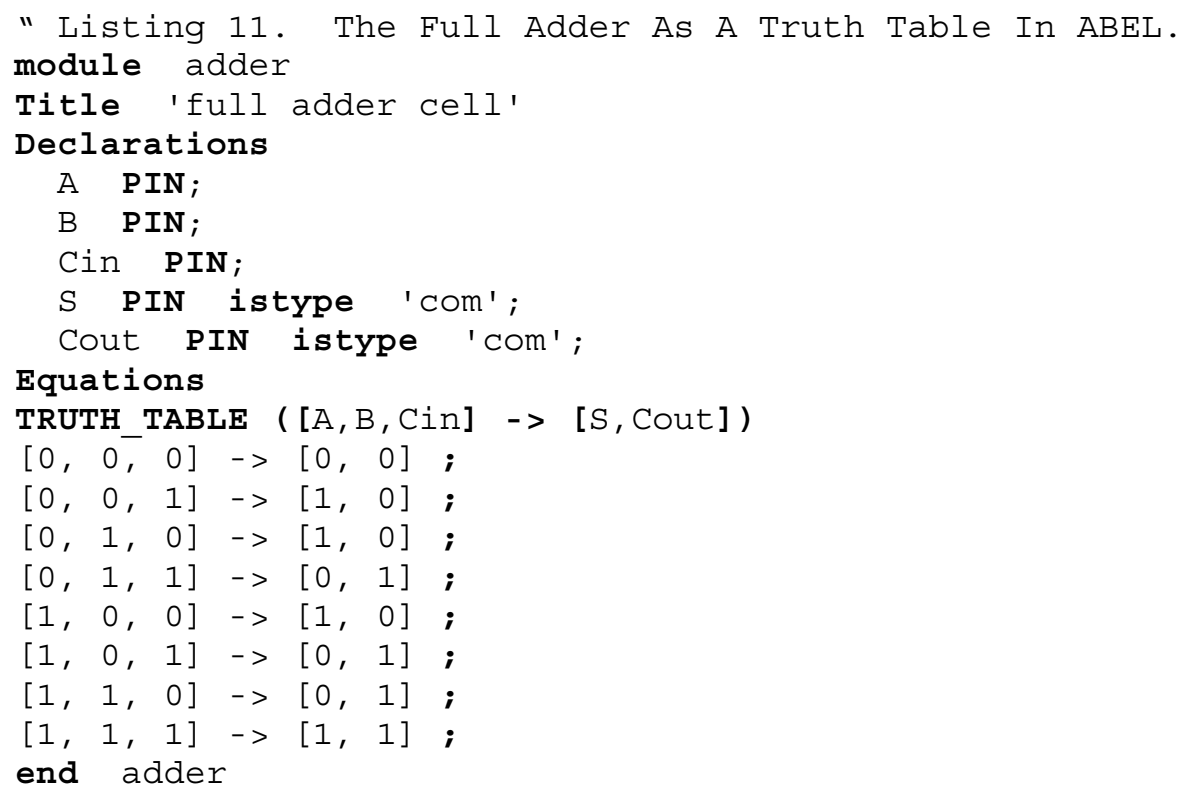

SDL appears simpler and cleaner, with less "fussy syntax" such as the repeated ->, [, ], and ; operators.

Comparisons With VHDL. SDL is simpler, but less powerful, than VHDL. SDL treats signals and variables identically, and does not explicitly distinguish between structural, dataflow, and behavioral modeling. SDL's default paradigm is structural, and in its 
schematic drawings it represents any behavioral or dataflow operations as black boxes. SDL does not recognize concurrent statements; therefore the order of statements is always significant. Component declarations are unnecessary, and component instantiations need not be named.

A few specific examples follow. First, a VHDL conditional dataflow description of a 4to-1 multiplexer ${ }^{10}$ is shown in Listing 12.

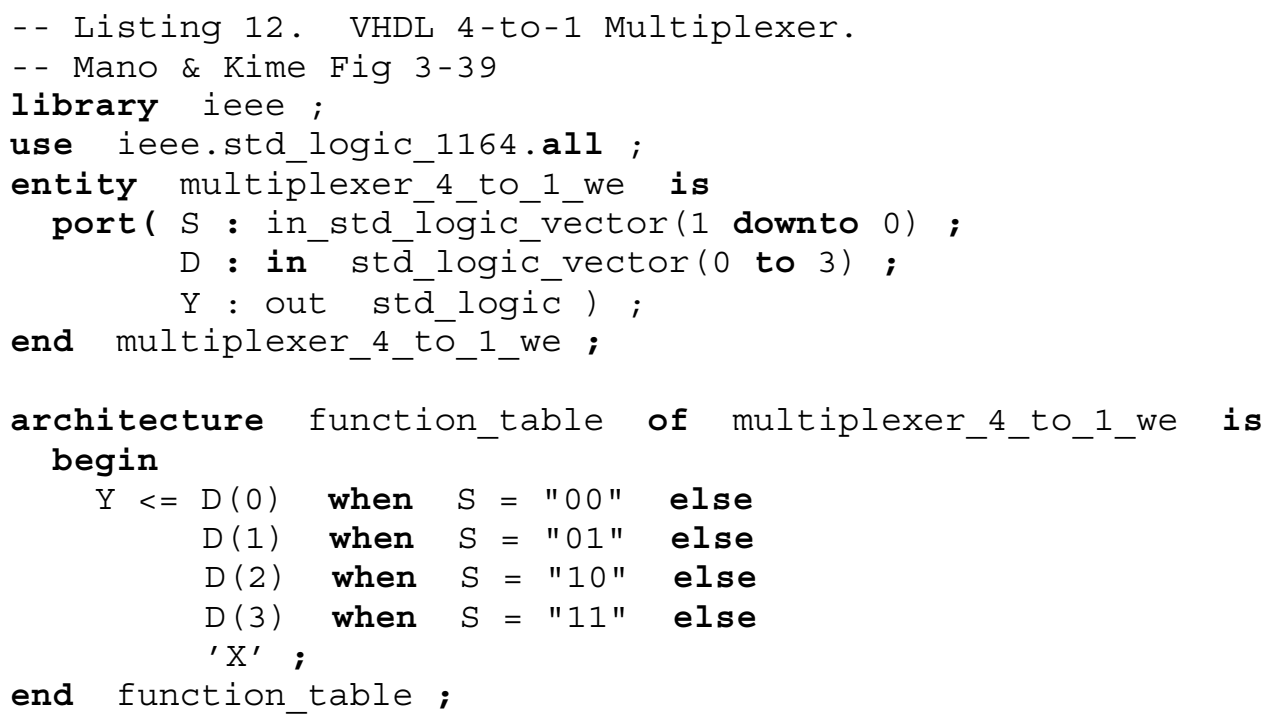

The corresponding SDL description is shown in Listing 13:

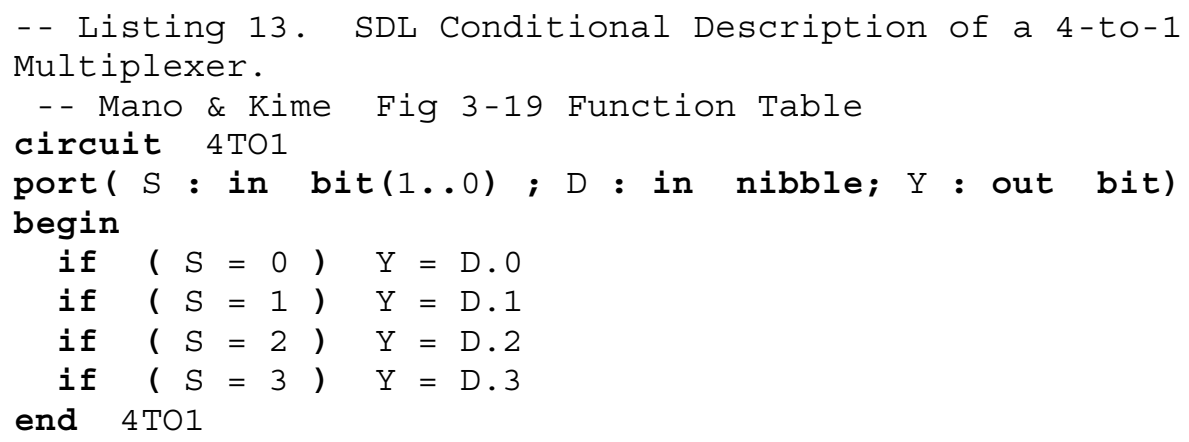

In each case, $D$ is a 4-bit variable. VHDL uses parentheses to refer to individual bits of a multibit variable (e.g., $\mathrm{D}(0), \mathrm{D}(1)$, etc.) while SDL uses a dot notation (e.g., D.0, D.1, etc.) . VHDL refers to an external library, while SDL is self-contained. This example uses VHDL's when...else construct, while SDL uses its if construct. The SDL file is small, clean, and relatively easy to explain to beginning students.

Listings 14 and 15 compare behavioral descriptions of a shift register in VHDL and in SDL.

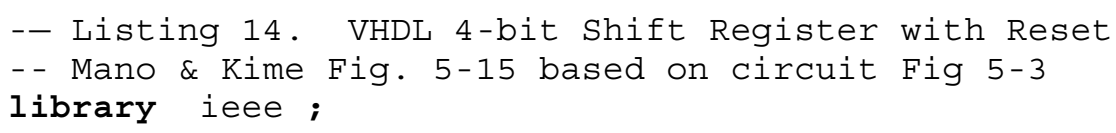

Proceedings of the 2001 American Society for Engineering Education Annual Conference \& Exposition Copyright @ 2001, American Society for Engineering Education 


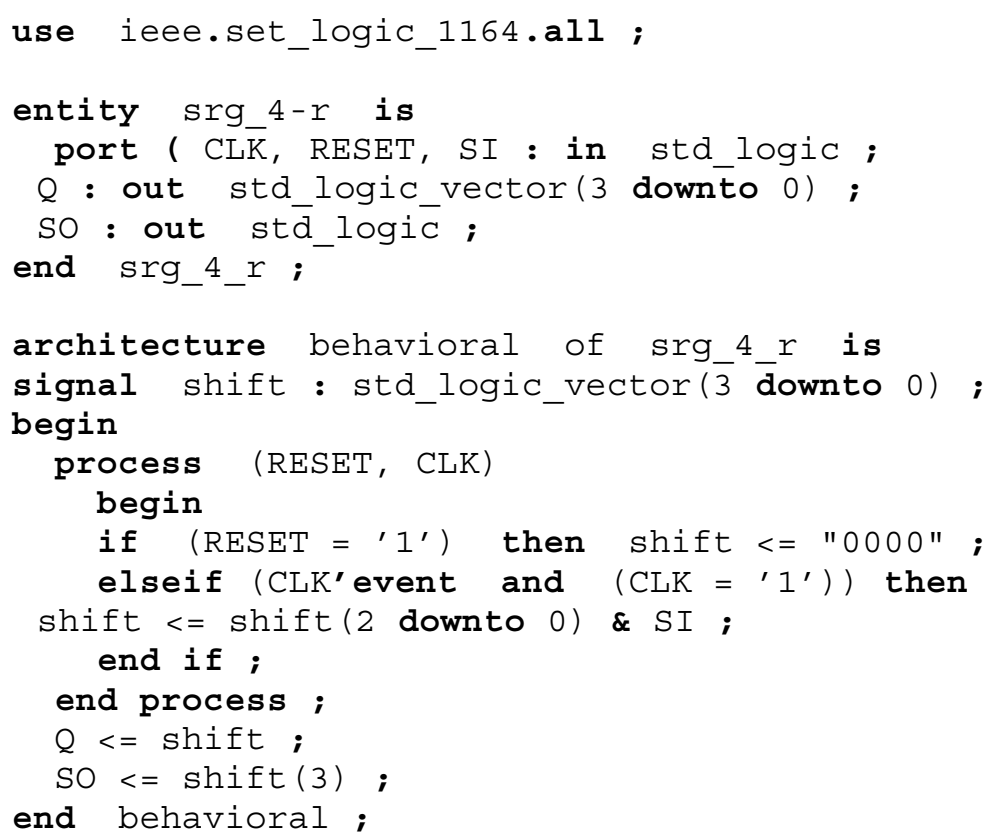

The SDL description is given in Listing 15:

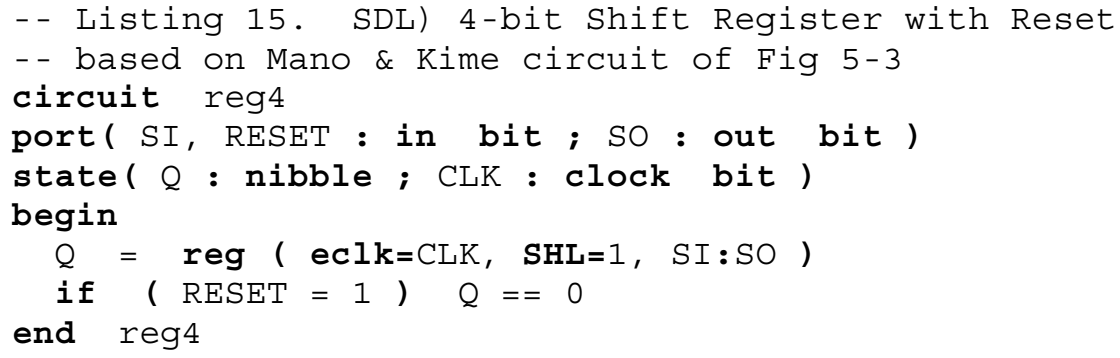

VHDL encodes the shifting operation in a process, whereas SDL includes it in its reg (register) function. VHDL uses the $<=$ operator for concurrent statements, SDL imposes concurrency by including all transfers within the same reg statement.

A common characteristic found in many comparisons of SDL descriptions with either ABEL or VHDL descriptions is that they are shorter. Certainly this is the case for the examples cited here, and they are not unrepresentative. Further SDL comparisons are available on request from the author ${ }^{11}$.

\section{Student Views.}

At the end of the Fall 2000 semester, I surveyed approximately 40 students in my two sections of the ELEC-229 course, and another 15 students in a third section taught by another professor. For the survey instrument (see Appendix) I adapted a form described in a previous ASEE paper ${ }^{12}$. Most questions were of the multiple-choice type, but the last three invited essay-type responses.

In the surveys, students described their experiences with digital simulation software, 
including SDL (55 students), VHDL (11 students), PSpice (33 students), and Electronics Workbench (1 student).

All students answered "Yes", they had used SDL. This is not surprising because we emphasized this simulation tool in our classes and in experiments. Most of the shortanswers were based on the scale of 1 (very successful) to 5 (not successful), with 0 a possible answer in one case. Any responses other than these six were categorized as "other" in the tabulations which appear in the Appendix.

Most of our students were taking their electrical engineering circuits course concurrently, and were exposed to PSpice through that venue. This accounts for most of the 33 students who claimed experience with PSpice. The responses show that some learned PSpice before SDL.

While we spent some class time discussing VHDL, and the textbook ${ }^{8}$ was bundled with a VHDL CD-ROM $^{13}$, we put more emphasis on understanding and using SDL. As a result, a relatively small number of students (11) claimed knowledge of VHDL.

The final survey question survey was: How would you characterize the role of SDL for you in course ELEC-229? Five possible check-box responses were included. Fifty-four students responded as follows:

-Useless (1 response)

- Confused me (8 responses)

- Helped me (37 responses)

- Just another thing to learn (12 responses)

- Other, please explain: ___ Only seven students weighed in on this invitation to essay:

Both confused and helped. Some circuits are harder than others. On the other hand, SDL does make it easier to understand the circuit if you take the time to go through all the commands and build the correct circuit.

In the beginning it helped me, but from the registers on it was just another thing to learn. It became more of a hassle to code right than to understand what the ckt is doing.

It was useful however not as user friendly as it could be.

Liability in the grade!

and it's easy to use.

Intuitive and very helpful

Very useful, but I haven't really known it to take advantage of its power.

A total of eight students had multiple answers and are included in more than one category.

When asked what they looked for in a new piece of software, over $50 \%$ of the respondents used the words "easy", "simple", or "not complicated", and over $25 \%$ used variations of the phrase "user-friendly".

\section{Conclusions.}

When a student begins to study a new subject, simplicity is paramount. There is no point in making her struggle with the peculiarities of a complex simulation program, while at the same time she is wrestling with the concepts of the course. If the program is not simple and intuitive, then it is just one more obstacle for her to overcome, and not a help 
in learning the course. As one of the responses to the student survey put it, "If it is too complicated, no one will bother to use it."

A simple program must not be overly "fussy" about syntax details, and it must be clear about any objections it has to syntax in a particular input file. As I was developing the program, I wrote many test files, and never once misspelled the keyword "circuit".

However, on the first day I had my class try their hands writing SDL circuit description files, one student did just that! To avoid user frustration, a "simple" program should report such errors back to the user with as much specificity and clarity as possible. Through student feedback, the program has evolved to become more user-friendly by providing clearer error messages, and suggestions for how to correct the error.

The SDL program represents an educational experiment in clarifying digital concepts for engineering students taking their first course in the subject. The language has an easier learning curve than VHDL and other well-established HDLs. I am satisfied that it is a helpful addition to the array of teaching tools available for the college professor. The program is available to anyone from Manhattan's website ${ }^{14}$, and I would welcome reports of additional experiences with it on other campuses.

Appendix

Students in Fall 2000 ELEC-229 were surveyed anonymously at the end of the semester, using a 1-page short-answer form. The questions, and a summary of responses to the non-essay questions, are given here. The numbers in each box show the counts of how many responses of 1 to 5 respectively were received. Numbers in parentheses indicate some other responses. Tabulations in the "Other" column are not included due to the low number of responses in that category.

\section{Survey on ELEC-229 Student Experiences With Digital Simulation Software}

\begin{tabular}{|l|c|c|c|c|}
\hline $\begin{array}{l}\text { Have you used any of the following digital } \\
\text { simulation software? } \\
\text { Y = Yes N = No }\end{array}$ & SDL & VHDL & PSPICE & Other \\
\hline $\begin{array}{l}\text { If more than one, indicate the order (1,2,3,4) } \\
\text { you've learned the software. }\end{array}$ & $448200(11)$ & 64100 & $723110(1)$ & $\mathbf{1 1}$ \\
\hline $\begin{array}{l}\text { How long did it take you to become } \\
\text { comfortable with the software? }\end{array}$ & 1525564 & $13123(1)$ & $1315031(1)$ & \\
$\begin{array}{l}\text { 1. One session. 2. Two weeks 3. Eight weeks. } \\
\text { 4. The entire semester. 5. Forever (never). }\end{array}$ & $11211543(1)$ & 00632 & $761150(4)$ & \\
\hline $\begin{array}{l}\text { Rate the difficulty of use for digital circuits. } \\
\text { Easy } 12345 \text { Difficult }\end{array}$ & 2118864 & $12223(1)$ & $1010840(1)$ & \\
\hline $\begin{array}{l}\text { How successful were you in starting the } \\
\text { software? } \\
\text { Very successful } 12345 \text { Not successful }\end{array}$ & 2016874 & $01432(1)$ & $78863(1)$ & \\
\hline $\begin{array}{l}\text { How successful were you in your first } \\
\text { simulation with this software? } \\
\text { Very successful 1 2 } 34 \text { Not successful }\end{array}$ & & & & \\
\hline
\end{tabular}




\begin{tabular}{|c|c|c|c|}
\hline $\begin{array}{l}\text { How successful are you in using the software } \\
\text { today? } \\
\begin{array}{lllllllll}\text { Very successful } & 1 & 2 & 3 & 4 & 5 & \text { Not successful }\end{array}\end{array}$ & 2121463 & $02323(1)$ & $139721(1)$ \\
\hline 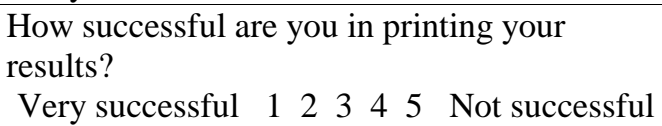 & 3611224 & $42121(1)$ & $212450(1)$ \\
\hline 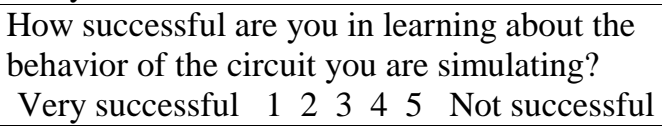 & 15231241 & $11340(2)$ & $138830(1)$ \\
\hline $\begin{array}{l}\text { How successful were you in installing the } \\
\text { software on your own computer? } \\
\text { 0. Did not try to install it. } \\
\text { Very successful } 1123445 \text { Not successful }\end{array}$ & 10383112 & $530110(2)$ & $13113211(2)$ \\
\hline $\begin{array}{l}\text { How successful are you in finding everything } \\
\text { you need in the online help? } \\
\text { Very successful } 1 \begin{array}{lllll} & 2 & 3 & 4\end{array} \text { Not successful }\end{array}$ & $1915863(4)$ & $21322(1)$ & $75843(6)$ \\
\hline
\end{tabular}

Is there any feature missing you would like this software to have? Specify which program you are writing about, if you have experience with more than one program.

What do you look for in a good piece of software?

How would you characterize the role of SDL for you in course ELEC-229?

[1 Useless [8] Confused me [37] Helped me [12] Just another thing to learn

[7] Other, please explain

\section{References}

1. MATLAB: The Language of Technical Computing, The MathWorks, Inc., Natick, MA, 1996.

2. Mathcad 2000 User's Guide, MathSoft, Inc., Cambridge, MA, 1999

3. Pellerin and Holley, Digital Design Using ABEL, Prentice Hall (1994)

4. Electronics Workbench, North Tonawanda, NY

5. Logic Works 4, Capilano Computing Systems, Ltd., New Westminster, Canada (1999)

6. Microsim Corporation, Laguna Hills, CA

7. Bhasker, A VHDL Primer, Prentice-Hall (1999)

8. Mano and Kime, Logic and Computer Design Fundamentals, Prentice-Hall, 2nd ed. (2000)

9. Digital Logic Design Principles, Balabanian \& Carlson, John Wiley \& Sons (2001), page 292

10. Mano \& Kime, op. cit., Figure 3-39 (page 154)

11. Approximately 13 pages (unpublished), available on request from the author.

12. Cooney and Monsanto, A Comparison of Electronic Design and Analysis Packages, ASEE National Conference, June 2000, Session 1620.

13. Xilinx Student Edition Foundation Series Software

14. http://www.engineering.manhattan.edu/ece/index.htm 


\section{ROBERT J. BORRMANN}

Robert J. Borrmann is Professor of Electrical and Computer Engineering at Manhattan College in New York City. He received the M.S.E.E. and Ph.D. degrees from the Polytechnic Institute of Brooklyn, and has taught extensively in the areas of electronics, computers, networks, and software engineering.

Proceedings of the 2001 American Society for Engineering Education Annual Conference \& Exposition 